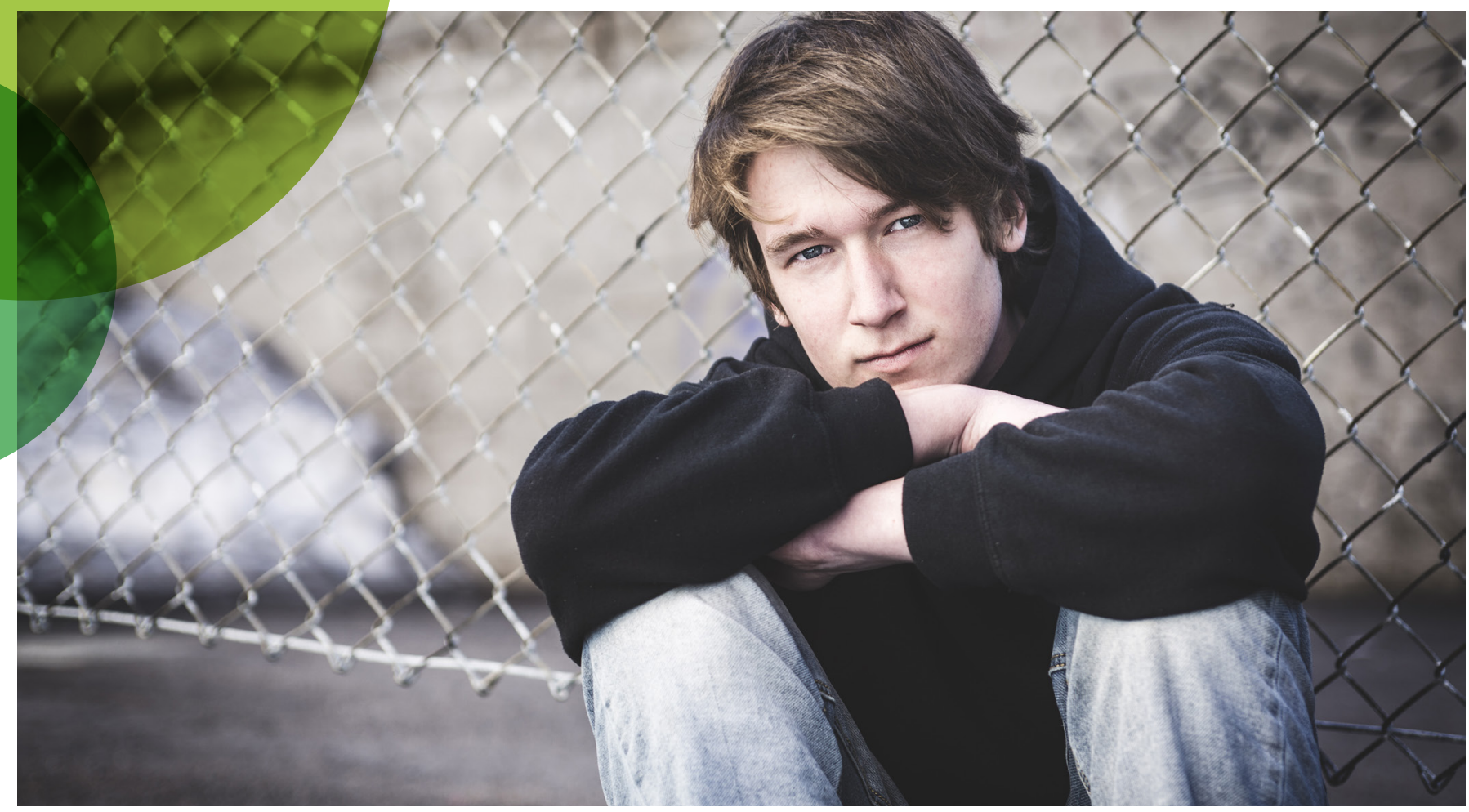

\title{
Early cognitive therapy for traumatised young people works and is also cost-effective
}

\section{By James Shearer}

James Shearer is a research health economist at the Institute of Psychiatry, Psychology and Neuroscience, Kings College London. His interests are the economics of mental health treatments for children and adolescents and using economic modelling to measure wider impacts on families, and the long term impacts on education and employment.

This article is a summary of the paper published in JCPP "Cost-effectiveness of cognitive therapy as an early intervention for post-traumatic stress disorder in children and adolescents: a trial based evaluation and model" Shearer, J., Papanikolaou, N., Meiser-Stedman, R., McKinnon, A., Smith, P., Dixon, C., Byford, S. (2018) Journal of Child Psychology and Psychiatry, 59 (7)): 773-780 doi: 10.1111/jcpp.12851

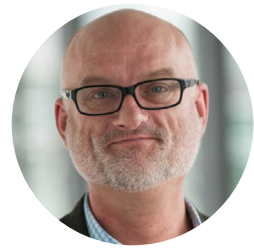

More than half of children and adolescents will experience traumatic events like vehicle accidents, house fires, or violence. However, brief counselling for young people in the immediate aftermath of an acute traumatic event has not be shown to be any more effective than not intervening and allowing natural recovery to take its course. Nevertheless, around $16 \%$ will go on to develop post-traumatic stress disorder (PTSD), a severe mental health condition with distressing and serious long-term symptoms such as intrusive memories, nightmares, problems concentrating and hypervigilance.

Untreated PTSD in early life is associated with other mental health problems, such as anxiety, depression and behavioural issues, and it has measurable economic consequences. These can include greater demand on NHS services, reduced quality of life for children and their parents, and problems in education leading to poorer employment and educational outcomes in later life. 
In our clinical trial (published in the Journal of Child Psychology and Psychiatry last year), we evaluated a different approach to early intervention for youth exposed to trauma. Rather than attempting to intervene in the first few days or weeks, we waited until at least two months had passed to work with children and adolescents with significant PTSD symptoms, as a number of young people will experience natural recovery in the first weeks following a trauma.

Young people aged between 8 and 17 years who had not recovered by the two-month point were welcomed into the trial and were randomised to receive cognitive therapy for PTSD or a 10-week waiting list for the same intervention. Cognitive therapy for PTSD involved 6-10 individual sessions focused on supporting children and adolescents to process their trauma. This therapy primarily involves helping them to understand their PTSD symptoms and why they have occurred, develop a coherent account of what occurred in the trauma, update their understanding of what happened (especially the most threatening or distressing aspects of what happened) and address behaviours that may be helping to maintain their symptoms.

In addition to collecting information on PTSD symptoms and other psychological outcomes, we also gathered information about any health, education and social care services used by the children during the study. We interviewed parents using a questionnaire specifically designed to collect this information, called the Child and Adolescent Service Use Schedule (CA-SUS).
The CA-SUS asks about their child's attendance at different types of school classes, absences from school or work, hospital-based treatments, A\&E attendances, medications and any contacts with a range of community-based health services like GPs and CAMHS workers. Our health economists then costed all of the services used by multiplying the number and duration of contacts by an appropriate unit cost.

The cost of the cognitive therapy was based on the number of contact hours multiplied by the hourly salary rate for a clinical psychologist Band 8a, factoring in overheads and non-contact time such as note writing, supervision, meetings etc. Overall, the children in the treatment group had an average of 636 minutes of contact time over 8 sessions, resulting in a value for the program of $£ 1,463$ per child, on average. We added this to the cost of the other services these children received and the total cost for the children who received cognitive therapy was $€ 1,691$ compared to E351 for the children who did not receive the therapy.

The next step in an economic evaluation is to look at the effects. In the UK, the main measure of effect used in economic evaluations is quality-adjusted life years (QALYs). QALYs weight survival time to reflect better or worse quality of life. For example, a year lived in perfect health equals I QALY but a year spent in less than perfect health is worth less depending on the severity of health problems. The lower the weight the worse the quality of life.

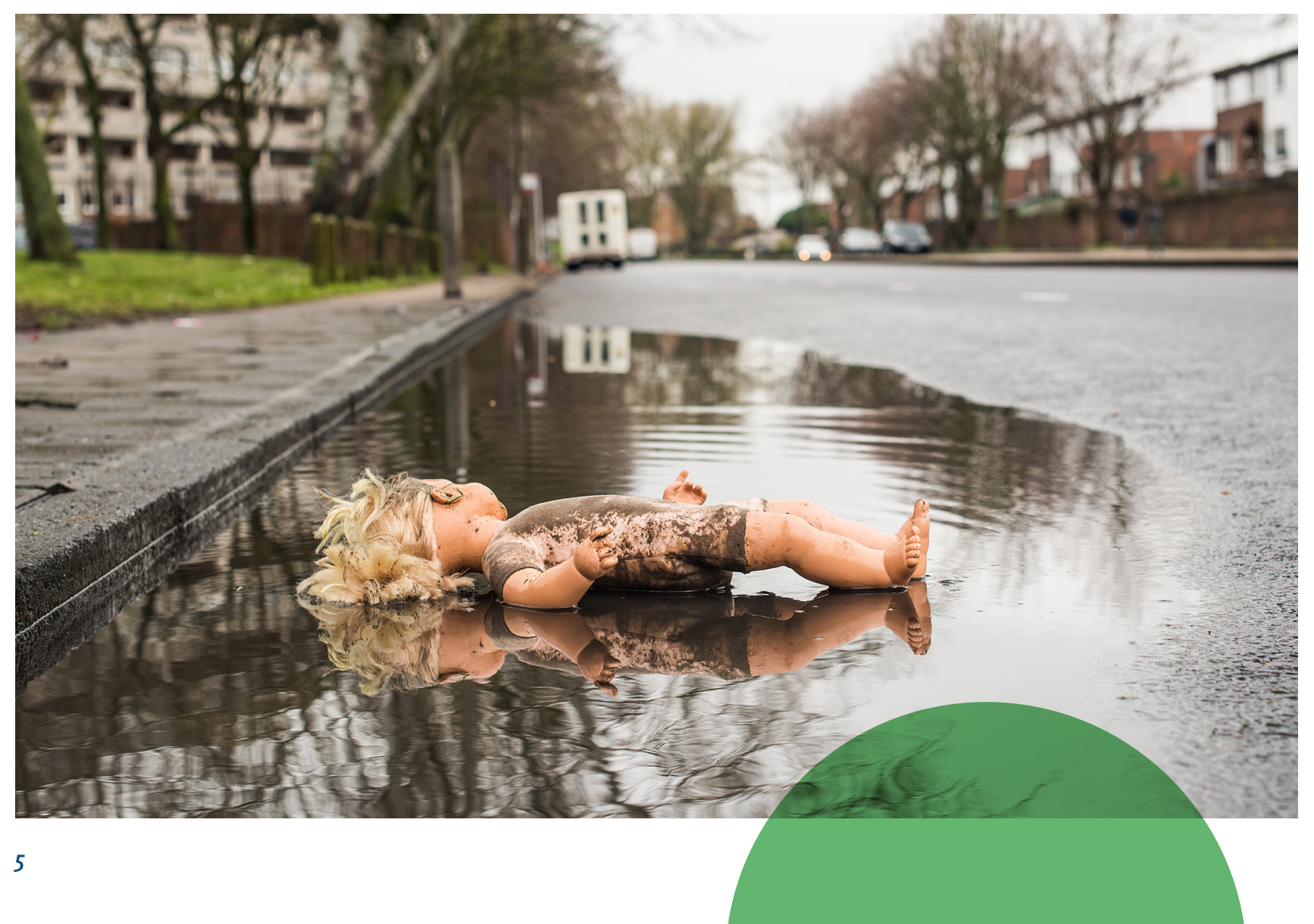


The children were not directly asked about their quality of life, instead we were able to estimate it from a clinical measure - the parent-completed Strengths and Difficulties Questionnaire (SDQ) - a widely used measure of mental health difficulties in young people. We used an equation which converted the SDQ score into a score on the Child Health Utility index (CHU$9 D$ ), a measure capable of generating QALYs. After 11 weeks, the children who received cognitive therapy had slightly more QALYs than those who did not.

Finally, we built a mathematical model, called a decision model, to forecast what would happen to the children up to 3 years after the study in terms of both their QALYs and costs. This was necessary because most of the benefits from treating PTSD will happen after the study has ended. The final result was that early cognitive therapy was estimated to be highly costeffective compared to the comparison group after three years, costing £2,205 for every additional QALY gained which is well below the National Institute for Health and Care Excellence (NICE) guidance of $£ 20,000$ to $£ 30,000$ per QALY for new therapies to be accepted as good value for money in the NHS.

Economic data can be very influential and this study has already been used to inform the development of NICE guidance in PTSD. The effort made to collect detailed service use and quality of life data has really paid off and we encourage future studies to add these types of measures. The economic modelling, though complicated, was also invaluable in helping to demonstrate the longer-term benefits of a successful but intensive, and therefore costly, therapy.

\section{Key points:}

What this evidence suggests is that early PTSD is worth taking seriously and treating. These sorts of early reactions (i.e. 2-6 months posttrauma) are not just transient distress, and we know that treatment like cognitive therapy for PTSD can lead to improvement in quality of life in a way that is cost-effective. It may help parents, hospitals, schools and colleges to know that effective and cost-effective interventions are available to help children and young people, and consider how they might help them to access this kind of therapy. Future research might also help us to understand what is cost effective for treating other forms of PTSD in children and young people, e.g. following multiple trauma exposure. For now, however, the evidence suggests that investing in supporting mental health professionals to deliver psychological therapies such as cognitive therapy for PTSD is worth it. 